Otto Hintze als Sozialtyp des Kaiserreichs. Neue Beiträge zu einem Berliner Gelehrtenprofil

In: Berichte und Abhandlungen / Berlin-Brandenburgische Akademie der Wissenschaften

(vormals Preußische Akademie der Wissenschaften) ; 16.2014, S. 103-122 


\section{Otto Hintze als Sozialtyp des Kaiserreichs. Neue Beiträge zu einem Berliner Gelehrtenprofil}

Otto Hintze (1861-1940) ${ }^{1}$ wurde im Frühjahr 1914, unmittelbar nach der Wahl Albert Einsteins, als Ordentliches Mitglied in die Preußische Akademie der Wissenschaften aufgenommen. ${ }^{2}$ Im Jahre 1933 war Hintze der einzige, „,der in der Akademiesitzung gegen Einsteins Ausschluß Einspruch erhob. Allgemeines Schweigen folgte seinen Worten. Erst während er in der Garderobe nachher seinen Mantel anzog, drückten einige ihm verstohlen die Hand."3 Und gut fünf Jahre später quittierte Hintze die Frage, ob er selbst ,jüdisch versippt“" sei, mit der Erklärung: ,Selbstverständlich lege ich meine Mitgliedschaft bei der Preuß. Akademie der Wissenschaften nieder."

Tragik liegt über Leben und Werk Otto Hintzes, dessen wissenschaftliches Erbe vor einem Halbjahrhundert, als in Geschichts- und Sozialwissenschaften ein Modernisierungsschub einsetzte, eine produktive Konjunktur erlebte. Seit den 1960er Jahren wurde Hintze als der „,wohl bedeutendste deutsche Historiker in der ersten Hälfte“

1 Obige Ausführungen beziehen sich ergänzend auf einen Vortrag des Verf. in der Geisteswissenschaftlichen Klasse: Otto Hintzes Weg zur Weltgeschichte, der in Saeculum, Jahrbuch für Universalgeschichte 2013, Heft 1 (im Druck 2014) erscheinen wird; es sei auf die dortigen Nachweise verwiesen, um den Apparat der vorliegenden Skizze zu entlasten.

2 Die Aktenstücke über die Aufnahme beider in die Akademie liegen in der einschlägigen Akte nur wenige Blatt voneinander entfernt: Archiv der BBAW, Historische Abteilung, Sign. II-III, 36: Personalia Mitglieder OM 1912-1916; vgl. nur noch Amburger, Erik: Die Mitglieder der deutschen Akademie der Wissenschaften zu Berlin 1700-1950. Berlin 1950, S. 37.

3 So die Mitteilungen bei Braun-Vogelstein, Julie: Was niemals stirbt. Gestalten und Erinnerungen. Stuttgart 1966, S. 352f.; vgl. damit Kirsten, Christa/Treder, Hans-Jürgen (Bearb.): Albert Einstein in Berlin 1913-1933, Teil 1. Berlin 1979, S. 265 (Nr. 191); Austritt: Ausgefüllter „Fragebogen“, ob Hintze u. a. ,jüdisch versippt“ sei, mit eigenhändiger Erklärung Hintzes vom 2. Dezember 1938, Archiv der Berlin-Brandenburgischen Akademie der Wissenschaften, Hist. Abt., II-III, 46. 


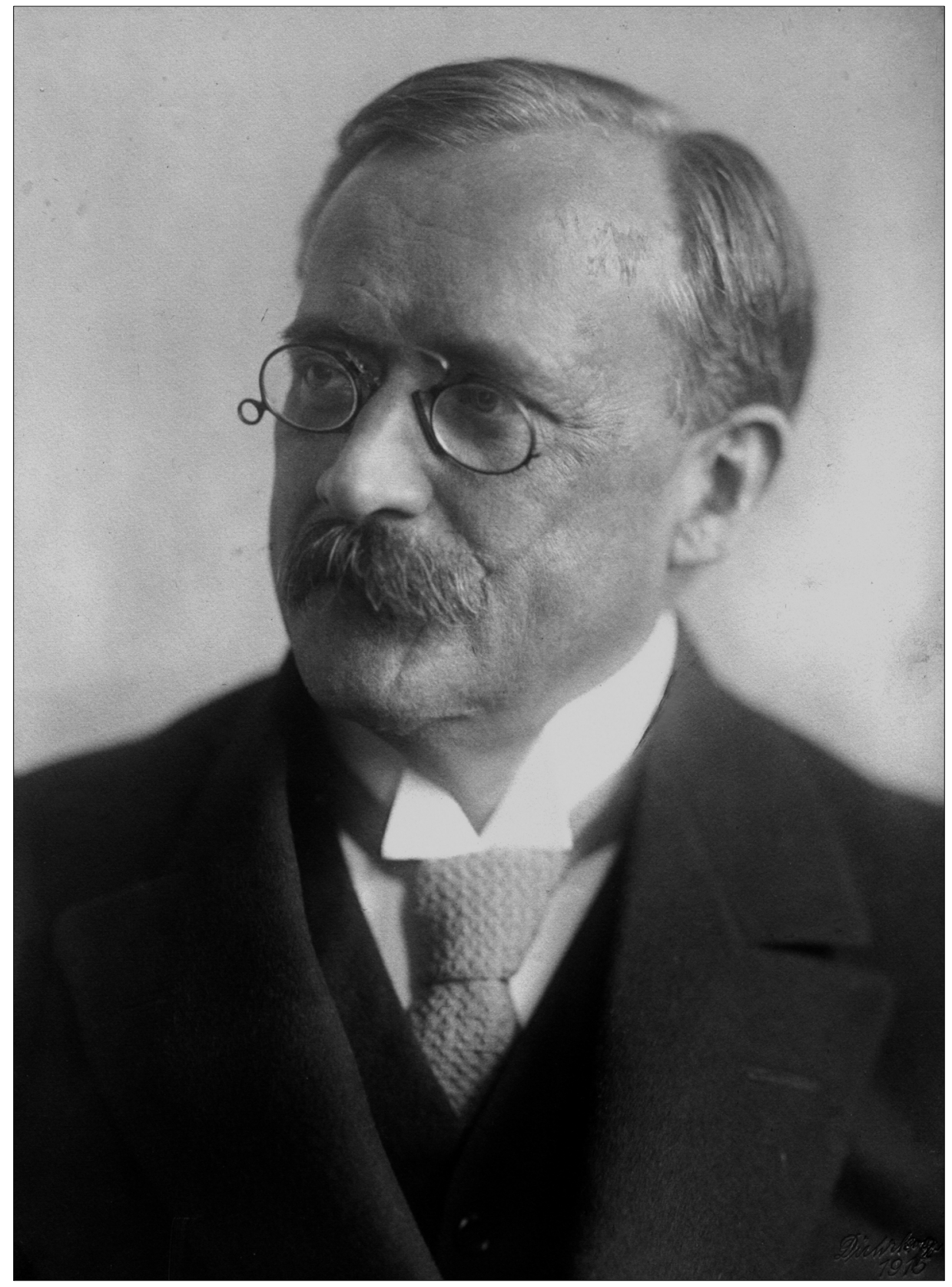

Otto Hintze

(Archiv der BBAW, Fotosammlung, Otto Hintze, Nr. 2)

104 Wolfgang Neugebauer 
des 20. Jahrhunderts ${ }^{4}$ in Parallele zu Max Weber analysiert und rezipiert. Und doch ist unlängst festgestellt worden, dass die „Stärken Hintzes“ auch „für die gegenwärtige soziologische Theorie noch nicht annähernd ausgeschöpft zu sein scheinen“". Hintzes Werk, anschlussfähig für moderne sozial- und strukturgeschichtliche Forschungen und auch für den kulturgeschichtlichen Turn der letzten Jahrzehnte, ${ }^{6}$ trat gleichwohl zuletzt in den Hintergrund des Interesses; er wurde, um Hans Joas zu zitieren, ,trotz seiner Nähe zur Soziologie in der deutschen Soziologie schmählich vernachlässigt“. ${ }^{7}$ Das lag auch daran, dass Max Weber die universaleren Perspektiven zu bieten schien. ${ }^{8}$ Weber wurde sehr viel breiter rezipiert, und nicht zuletzt: Er wurde nun und auf höchstem Niveau ediert, während das Lebensschicksal Otto Hintzes zum Schicksal seines Euvres geworden ist. Seine Hauptwerke sind nicht erschienen, nicht zu Lebzeiten und auch nicht postum. Im Kriegsberlin des Frühjahrs 1940 hat Otto Hintze wenige Tage vor seinem Tode am 25. April eigenhändig testamentarisch verfügt, alle ,persönlichen Manuskripte“ zu vernichten. ${ }^{9}$ So sind die beiden Hauptwerke Hintzes, eine „Allgemeine und vergleichende Verfassungs- und Verwaltungsgeschichte der neueren Staatenwelt" und - vielleicht noch wichtiger eine systematisch angelegte „Allgemeine Staats- und Gesellschaftslehre“"10 (wohl

4 So Wehler, Hans-Ulrich: Einleitung. In: Ders. (Hg.): Moderne deutsche Sozialgeschichte. Köln ${ }^{4} 1973$, S. 9-16, hier S. 11 (= Neue Wissenschaftliche Bibliothek, Bd. 10. Geschichte). Hintze und Weber: als Beispiel Mommsen, Wolfgang J.: Einleitung. In: Ders./ Schwentker, Wolfgang: Max Weber und seine Zeitgenossen. Göttingen, Zürich 1988, S. 11-38, hier S. 26 (= Veröffentlichungen des deutschen Historischen Instituts London, Bd. 21).

5 So Joas, Hans: Gibt es eine militaristische Tradition in der Soziologie? In: Ders.: Kriege und Werte. Studien zur Gewaltgeschichte des 20. Jahrhunderts. Weilerswist 2000, S. 204-235, hier S. 233, wiederum mit gleichzeitigem Hinweis auf Max Weber.

6 Schorn-Schütte, Luise: Historische Politik-Forschung. Eine Einführung. München 2006, S. $37 \mathrm{f}$.

7 Joas, Hans: Die Modernität des Krieges. Die Modernisierungstheorie und das Problem der Gewalt. Zuerst 1996, wieder in: Ders.: Kriege (wie Anm. 5), S. 67-86, hier S. 81, Anm. 21.

8 In diesem Sinne Kocka, Jürgen: Otto Hintze, Max Weber und das Problem der Bürokratie. In: Historische Zeitschrift 233 (1981), S. 65-105, hier S. 65, S. 81f., S. 85.

9 Vgl. mit Hinweisen auf ungedruckte Quellen: Neugebauer, Wolfgang: Zur Quellenlage der Hintze-Forschung. In: Jahrbuch für die Geschichte Mittel- und Ostdeutschlands 45 (1999), S. 323-338, hier S. 324f. - und passim.

10 Seine programmatischen Mitteilungen in: Hintze, Otto: Rezension zu: Rudolf Kjellén, Grundriß zu einem System der Politik, 1920. In: Schmollers Jahrbuch 45 (1921), S. 266-271, hier S. 268, als wichtige Ergänzung zu seiner Antrittsrede in der Preußischen Akademie der Wissenschaften, zuerst 1914, wieder in: Hintze, Otto: Staat und Verfassung. Gesam- 
im Jahre 1942) untergegangen. Nur Fragmente aus der „Verfassungsgeschichte“ sind der Vernichtung entgangen und erhalten. Aber Hintze hat beide Werke mehrmals in Form von Vorlesungen vorgetragen, und wir sind in der glücklichen Lage, so wenigstens eine mündlich vermittelte Hilfsüberlieferung zu besitzen, die tiefe Blicke in seine Denkwelten zulässt. Die Auswertung dieser mehrere tausend Seiten umfassenden handschriftlichen Quellenmasse, darunter auch mehrere, sich gegenseitig ergänzende und kontrollierende Fassungen der ,Allgemeine(n) Staats- und Gesellschaftslehre" aus dem Jahre 1911, ${ }^{11}$ wird erst zeigen, wie verengt, ja fehlgeleitet die bisherige Rekonstruktion des Hintze'schen Erbes auf der Basis nicht sehr glücklich konstruierter Sammlungen seiner Aufsätze ${ }^{12}$ gewesen ist. Tatsächlich ging Hintzes Sicht von Geschichte, von Welt-Geschichte, schon bald nach 1900 auf globale Konzeptionen aus, verblasste der traditionale Typ des landgestützten Machtstaats (Preußen z. B.) zugunsten einer Perspektive kommender transatlantischer politischer Kulturen.

Prominenz besaß Hintze im Fach, nicht im breiten Publikum, und immer ist er auch Außenseiter geblieben, so wie er dies 1933 noch einmal aufrecht unter Beweis gestellt hat. Diese Position im Fach und das Schicksal seines Werkes sind ganz wesentlich in seiner Biographie begründet. Lange schien es, nach dem Verlust des ganzen BriefNachlasses, ja nach dem Untergang seiner Lebenswelt, gar nicht mehr möglich, seine Biographie zu schreiben. Nur zu weniges schien zu der Person Otto Hintzes überliefert. ${ }^{13}$ Gewiss gilt auch für ihn, dass die Methoden der Biographik von der in diesem Falle aber doch gar nicht so desaströsen - Quellenlage abhängig sind. ${ }^{14}$ Biographie und Werk, zusammen betrachtet, werden dann freilich zeigen, dass Hintzes Wirksamkeit, seine Weiten und seine Begrenzungen, ganz wesentlich sozial-

melte Abhandlungen zur Allgemeinen Verfassungsgeschichte, hg. von Oestreich, Gerhard. Göttingen ${ }^{3}$ 1970, S. 563-566, hier S. 564 und 566 zu dem damals noch als ,Staatslehre“ gedachten (2.) Hauptwerk; zum Folgenden: Hintze, Otto: Allgemeine Verfassungs- und Verwaltungsgeschichte der neueren Staaten. Fragmente, Bd. 1, hg. von di Costanzo, Giuseppe/Erbe, Michael/Neugebauer, Wolfgang. Neapel 1998, darin Neugebauer, Wolfgang: Otto Hintze und seine Konzeption der „Allgemeinen Verfassungsgeschichte der neueren Staaten“, S. 35-83, erweiterter und ergänzter Neudruck einer Studie von 1993.

11 Manuskript: „Prof Hintze SS 1911. Allgemeine Staats- und Gesellschaftslehre“, 4 Hefte, von (dem späteren Archivar) Ewald Guttbier; heute im Besitz des Verf.

12 Vgl. Anm. 10; die neue Sicht: wie Anm. 1.

13 So noch Oestreich, Brigitta: Einleitung. In: Dies. (Bearb.): Otto Hintze und Hedwig Hintze. Die Korrespondenz 1925-1940, hg. von Jütte, Robert/Hirschfeld, Gerhard. Essen 2004, S. 15-39, hier S. 17 (= Schriften der Bibliothek für Zeitgeschichte, NF, Bd. 17).

14 Mit allgemeiner Gültigkeit: Szöllösi-Janze, Margit: Fritz Haber 1868-1934. Eine Biographie. München 1998, S. 10f., s. auch unten Anm. 72. 
geschichtlich begründet sind. Die Kontextualisierung der Wissenschaftlerbiographie ist heute eine methodische Forderung, und das heißt im Falle Otto Hintzes, seine Prägung als wissenschaftsbürgerlicher Aufsteiger im kaiserreichlichen PreußenDeutschland als Faktor der Werkgenese ernst zu nehmen.

Otto Hintze kam von außen, nicht aus großbürgerlicher oder bildungsakademischer Familie, und er kam nicht aus einer urbanen Umwelt. ${ }^{15}$ Die Familie stammte aus Hinterpommern, und hier, im 6.500 Einwohner zählenden Pyritz, einer Stadt mit intakter mittelalterlicher Ummauerung, aber noch ohne Bahnanschluss und ohne Industrie, wurde Hintze am 27. August 1861 als Sohn des dortigen Kreissekretärs gleichsam Büroleiter des dort immer noch adligen Landrats ${ }^{16}$ - geboren ${ }^{17}$ Für einen bestenfalls mittleren Beamten muss es eine strategische Entscheidung gewesen sein, alle fünf Söhne, darunter Otto als Zweitgeborenen, auf das Humanistische Gymnasium der Stadt gehen zu lassen, schon dies eine schwere finanzielle Last, um sie alle nach Schul- und Schulgeldzeit auch noch studieren zu lassen. Der entschlossene Aufstiegswille der Familie kam auch darin zum Ausdruck, dass nicht etwa zunächst der Pfarrerberuf als Mobilitätskanal gewählt, sondern sogleich weitergehende Karrieren angezielt wurden. Unter Ottos Brüdern waren denn auch erfolgreiche Juristen und Mediziner, und er selbst hat seine Gymnasialzeit maximal komprimiert, um, gerade einmal 17 Jahre alt, mit dem damals nur von wenigen Gymnasiasten angestrebten Abitur abzugehen ${ }^{18}$, und zwar um „Philologie“ zu studieren. Beim Fehlen aller Familienpapiere wird man aus diesem Befund doch schließen dürfen, dass familiäre Aufstiegsstrategie und individueller Karriereplan sich gegenseitig stimulierten. Nach der Schulentlassung, am Sedanstag 1878, lag es nahe, zunächst die pommersche Landesuniversität Greifswald zu erproben, aber der dortige Unterricht im jungen historischen Seminar hat Hintze nicht sehr gefesselt, so dass nach vier nicht eben ergiebigen Semestern der schon einigermaßen mutige Entschluss gefasst worden ist, den Sprung nach Berlin zu wagen.

15 Die folgenden biographischen Ausführungen fassen Befunde aus einem weit verstreuten Quellenmaterial zusammen, die in einer im Entstehen befindlichen Biographie Otto Hintzes im Einzelnen nachgewiesen werden.

16 Vgl. Wagner, Patrick: Bauern, Junker und Beamte. Lokale Herrschaft und Partizipation im Ostelbien des 19. Jahrhunderts. Göttingen 2005, S. 76.

17 Lebenslauf von Otto Hintzes Hand aus dem Jahre 1899: Geheimes Staatsarchiv Preußischer Kulturbesitz (zit.: GStA PK), VI. Hauptabt., Nachlass Gustav Schmoller Nr. 231; vgl. ferner Hintzes Personalakte: Humboldt-Universität zu Berlin, Archiv, Bestand: Verwaltungsdirektor, Personalakte Lit. H 332, Bd. 2.

18 Nach dem Pyritzer Gymnasial-Programm dieser Jahre, hier speziell: 20. Programm des Gymnasiums der Stadt Pyritz [...], Pyritz 1879, S. 20; zum folgenden s. Anm. 15. 
Aus der Wohnsoziologie der Stadt um und nach 1880 und den Adressen des Studenten Otto Hintze ließe sich genauer ablesen, mit welcher verzweifelten Entschlossenheit der Griff nach der Hauptstadtuniversität unternommen worden ist: Berlin um jeden Preis, und wenn auch zunächst nur ein Unterkommen im ominösen Scheunenviertel im Alt-Berliner Norden möglich war, in Sichtweite dunkle Gassen und Gestalten, mit Kriminalität und Prostitution. Hintzes sehr lebendiges Wohnitinerar, sein Wohnhabitus, bis hin zur feudalen Kurfürstendamm-Wohnung seiner Ordinarienjahre, ist eine noch unausgeschöpfte Quelle von sozialgeschichtlicher Aussagekraft, nicht nur für diese Gelehrtenbiographie.

Halten wir inne: Selbstverständlich war das alles nicht, zumal die Quellen aus der Studienzeit Hintzes den Schluss nahelegen, dass trotz Probejahr am Berliner Friedrichsgymnasium nicht eigentlich eine mittlere Sozial-Karriere angestrebt worden ist. ${ }^{19}$ Die Hochschullehrerlaufbahn war aber in dieser Zeit eine ausgesprochene „Domäne des gehobenen Bürgertums“; im 19. Jahrhundert stammten im Durchschnitt über 72 Prozent der deutschen Professoren aus der ,,höheren Beamtenschaft“, aus Familien des Besitzbürgertums und der freien Berufe. ${ }^{20}$ Insofern war die soziale Herkunft Otto Hintzes gar nicht so typisch, sondern sie verweist auf die Zugehörigkeit zu der immerhin sehr wohl vorhandenen Minderheit von Aufsteigern im System von Stellung und Sozialprestige. Pierangelo Schiera hat darauf aufmerksam gemacht, dass trotz beachtlicher vertikaler Mobilität unter denen, die akademische Grade erlangten, seit etwa 1890 bei den Habilitanden der großbürgerliche Einschlag quantitativ an Gewicht gewann. „Für den [...] kleinen Beamten mag es wohl typischer gewesen sein, den Sohn Oberlehrer oder mittleren Beamten werden zu lassen, als sofort auf den höheren Beamten oder den Professor zu zielen“. ${ }^{21}$ Um 1910 stammten „2 \% der

19 Mit Nachweisen: Neugebauer, Wolfgang: Otto Hintze (1861-1940). In: Fröhlich, Michael (Hg.): Das Kaiserreich. Portrait einer Epoche in Biographien. Darmstadt 2001, S. 286-298, hier S. 286f., S. 295 Anm. 2, und Humboldt-Universität zu Berlin (wie Anm. 17).

Nach Baumgarten, Marita: Professoren und Universitäten im 19. Jahrhundert. Zur Sozialgeschichte deutscher Geistes- und Naturwissenschaftler. Göttingen 1997, S. 110 (= Kritische Studien zur Geschichtswissenschaft, Bd. 121), die Hervorhebung im Zitat von W. N.; das Folgende: Schiera, Pierangelo: Laboratorium der bürgerlichen Welt. Deutsche Wissenschaft im 19. Jahrhundert. Frankfurt a. M. 1992, S. 293.

21 So Ringer, Fritz: Das gesellschaftliche Profil der deutschen Hochschullehrerschaft 1871-1933. In: Schwabe, Klaus (Hg.): Deutsche Hochschullehrer als Elite 1815-1945. Boppard a. Rh. 1988, S. 93-104, Zitat: S. 102 (= Deutsche Führungsschichten in der Neuzeit, Bd. 17); das Folgende: Ringer, Fritz: Die Gelehrten. Der Niedergang der deutschen Mandarine 1890-1933. Stuttgart 1983, S. 61. Die Zahlen differieren in tolerierbaren Grenzen, vgl. etwa Jarausch, Konrad H.: The Transformation of the University: The Case 
Universitätsstudenten Preußens aus Arbeiterhaushalten und lediglich 3 \% waren Söhne von kleinen (nicht leitenden) Angestellten“(Ringer).

Fragen wir spezifischer nach der sozialen Herkunft deutscher Hochschullehrer in der Zeit des Kaiserreichs, so lag der Anteil der Söhne „kleiner Beamter“ bei 5 Prozent gegenüber denjenigen aus der „traditionellen Bildungsschicht“ mit um die 60 Prozent, um 1900 dann 49 Prozent. Es dominierte die Basis privaten Vermögens. Aber im Vergleich etwa zu England und Frankreich war die Herkunft der Universitätsprofessoren „durchaus nicht extrem exklusiv“, sondern offen für „Außenseiter“ und Aufsteiger (Mommsen). ${ }^{22}$

Die These lautet, dass diese typologische Stellung des Kreissekretärsohnes Otto Hintze aus Pyritz/Pommern von werk- und damit wissenschaftsgeschichtlicher Relevanz gewesen ist. Immerhin: Der mutige Schritt des Jahres 1880 trug alsbald Früchte. In Berlin fand Hintze rasch Zugang zur akademischen Prominenz, zumal zu Person und auch zum Haus von Johann Gustav Droysen, den er später einmal seinen „hochverehrten, unvergeßlichen Lehrer“ genannt hat, ${ }^{23}$ auch wenn er nicht bei ihm (zu einem Thema des 13. Jahrhunderts) promovierte. Im Berlin der 1880er Jahre erhielt er die entscheidenden intellektuellen Impulse, solche, die ihm schon früh moderne Fragestellungen nahebrachten, die die Forschung wohl zu Unrecht erst in seinem späteren Werk aufsucht. Denn schon bei seiner Promotion, mit gut 22 Lebensjahren 1883/84, finden sich Spuren von Anregungen, die er erst in seinen Berliner Semestern empfangen hatte. Wollte Hintze doch bei seiner Promotion außer über die Frage, wie Geschichte zur ,selbständigen Wissenschaft“ werden könne, auch schon über das Verhältnis von Staat und „Gesellschaft“ disputieren: „Der Begriff des Staates ist als der primäre dem der Gesellschaft vorauszustellen“ - so lautete damals seine These. ${ }^{24}$ Damit waren schon bei dem Kandidaten Hintze Grundprobleme (nicht nur der Geschichtswissenschaften) formuliert, die ihn in der Tat lebens-

of Prussia 1865-1914. In: Journal of Social History 12 (1979), S. 609-636, hier S. 625, Plutokratisierungstendenz im späten 19. Jahrhundert: S. 627.

22 Mommsen, Wolfgang J.: Bürgerliche Kultur und künstlerische Avantgarde. Kultur und Politik im deutschen Kaiserreich 1870-1918, Studienausgabe Frankfurt a. M. 1994, S. 70, S. 73; Ringer: Profil (wie Anm. 21), S. $96 f$.

Brief Hintzes an Droysens Sohn, den Hallenser Professor Gustav Droysen (in der Universitäts- und Landesbibliothek Sachsen-Anhalt, Halle (Saale), Abt. Sondersammlungen, Nachlass Gustav Droysen, Sign. Yi 32 III H. 53-59), vom 19. August 1899.

24 In den nur dem Dissertationsdruck beigefügten „Thesen“; Hintze, Otto: König Wilhelm der Holländer. Ein Beitrag zur Geschichte des deutschen Interregnums (Einleitung und fünf Excurse). Phil. Diss. Berlin 1884, S. 35. Dazu die Akte im Archiv der HumboldtUniversität, [H 263], Promotionen, Phil. Fak. 1884. 
lang beschäftigen sollten, wenn sich auch sehr bald die Gewichte dieser beiden Kategorien Gesellschaft und Staat verschoben.

Zunächst schloss der junge Doktor ein Zweitstudium an. „Seit dem Wintersemester $1885 / 6$ an hiesiger Universität aufs neue immatrikuliert, habe ich mich zur tieferen Begründung meiner historischen Bildung dem Studium der Jurisprudenz und der Staatswissenschaften zugewandt" ${ }^{25}$ Zugleich verwies er schon im Frühjahr 1887 als Referenz auf den Herrn Professor Schmoller, der unter den Lehrern des Erststudiums noch gefehlt hatte. ${ }^{26}$

Damit zeichnet sich nun diejenige Konstellation ab, die Hintzes wissenschaftlichen Aufstieg ermöglichte und auch bedingte. Die ,systematisch-ökonomischen Interessen“ haben den jungen Otto Hintze zeitweilig ganz „ernsthaft an den Übergang zur Nationalökonomie [...] denken lassen““ ${ }^{27}$ So lag es nahe, zu dem Staatswissenschaftler und Nationalökonomen Gustav Schmoller Kontakt zu gewinnen, als der gerade - im Frühjahr 1887 - daran ging, das staatswissenschaftlich angelegte Editions- und Forschungsprojekt der Acta Borussica bei der Preußischen Akademie der Wissenschaften zu begründen, das der Staatsstruktur und der Wirtschaftspolitik Preußens im 18. Jahrhundert gewidmet wurde. ${ }^{28}$ Zunächst schwankte Schmoller noch, ob Hintze der geeignete Mitarbeiter sein würde; es war, wenn es so gefasst werden darf, keine wissenschaftliche Zuneigung auf den ersten Blick. Mancherlei Probearbeiten Hintzes waren 1887 erst zu leisten, darunter eine von entscheidender, weichenstellender Bedeutung für die Karriere Otto Hintzes im kaiserzeitlichen Berlin: Friedrich Althoff, damals noch am Anfang seiner fünfundzwanzigjährigen Regentschaft über die Wissenschaften (nicht nur) in Preußen, hatte in eben diesem Jahre Otto Hintze kennengelernt, als dieser dem mächtigen Vortragenden Rat im Kultusministerium $^{29}$ auf Vermittlung Schmollers eine Zuarbeit zu liefern hatte.

25 Diese Selbstbeschreibung seines Bildungsganges in einer eigenhändigen Eingabe Hintzes vom 24. März 1887: GStA PK, I. HA, Rep. 178, V, Nr. 6. Lit. H, Vol. 2.

26 Hintze: König Wilhelm (wie Anm. 24), S. 34.

27 Diese biographisch interessante Mitteilung in der Rezension von Brinkmann, Carl, zu: Otto Hintze: Gesammelte Abhandlungen, 2. Bd. [...] 1942. In: Historische Zeitschrift 169 (1949), S. 406f., hier S. 407.

28 Mit allen wesentlichen Details und hier nicht zu skizzierenden Hintergründen: Neugebauer, Wolfgang: Zum schwierigen Verhältnis von Geschichts-, Staats- und Wirtschaftswissenschaften am Beispiel der Acta Borussica. In: Kocka, Jürgen (Hg.): Interdisziplinäre Arbeitsgruppe Berliner Akademiegeschichte im 19. und 20. Jahrhundert. Die Königlich Preußische Akademie der Wissenschaften zu Berlin im Kaiserreich. Berlin 1999, S. 235-275.

29 Mit der inzwischen reichhaltigen Literatur: Spenkuch, Hartwin: Die Politik des Kultusministeriums gegenüber Wissenschaften und Hochschulen. In: Berlin-Brandenburgische 
„Hr. Dr. Hintze hat mir gut gefallen“ ${ }^{30}$ so lautete Althoffs Note, und wie es scheint, sind sich der preußische Universitätsbeherrscher und der junge Noch-Student Dr. Hintze schon zur Jahreswende 1887/88 mehrmals persönlich begegnet.

Diese Dreierkonstellation, Althoff, Schmoller und Hintze, hat für den pommerschen Historiker, Staats- und Wirtschaftswissenschaftler den Aufstiegsweg vorgezeichnet, freilich auch - was erst im Licht neuester Quellenfunde erkennbar ist - das Lebenswerk Otto Hintzes (de)formiert.

Zunächst allerdings sah dies alles doch günstig aus für den jungen Mann, der ganz offenbar ohne irgend familiäres Vermögen, angestellt beim Akademieprojekt der Acta Borussica, ${ }^{31}$ nun erst Bände zur preußischen Seidenindustrie im 18. Jahrhundert, dann zur preußischen Staatsorganisation und Behördenstruktur nach 1740 edierte und dazu zwei auch heute noch grundlegende Monographien schrieb, gefördert und gefordert von Schmoller bis hin zu gefährlichen physischen Zusammenbrüchen schon seit diesen frühen Jahren. Auch Althoff hat Hintze ab und zu wieder beschäftigt; es gibt Belege, dass dieser zum ministerialrätlichen Nachrichtenapparat gehörte und als Mitarbeiter nicht des Amtes, sondern der Akademie gut verwendbar war zum Einholen von Berichten über die Lehr- und Arbeitsweise außerpreußischer, etwa bayerischer Professoren, zum Beispiel über deren Vorlesungen. Gutachten hat Hintze für Althoff mehrmals geschrieben, eher historisch interessante, wie eines ,über die hohenzollerschen Universitätsgründungen“. ${ }^{32}$ Das waren offenbar umfangreiche Arbeiten, und so wurde überlegt, ob daraus nicht ein zu publizierendes „Werk“ wer-

Akademie der Wissenschaften (Hg.): Acta Borussica. Neue Folge, 2. Reihe: Preußen als Kulturstaat, Abt. I, Bd. 2.1. Berlin 2010, S. 135-287, bes. S. 173-176; Lüdicke, Reinhard (Bearb.): Die Preußischen Kultusminister und ihre Beamten im ersten Jahrhundert des Ministeriums 1817-1917. Stuttgart, Berlin 1918, S. 29f., S. 88.

Nach der Korrespondenz zwischen Friedrich Althoff und Gustav Schmoller im Jahre 1887, zunächst: GStA PK, VI. HA, Nachlass Althoff, A II, Nr. 95 II, und Nachlass Schmoller, Nr. 112, hier das obige Zitat im Brief Althoffs vom 10. Dezember 1887; vgl. detaillierter Neugebauer, Wolfgang: Die wissenschaftlichen Anfänge Otto Hintzes. In: Zeitschrift der Savigny-Stiftung für Rechtsgeschichte. Germ. Abt. 115 (1998), S. 540-551, worauf ich mich für das zunächst Folgende beziehe.

31 Die Quellen ediert bei Neugebauer, Wolfgang: Gustav Schmoller, Otto Hintze und die Arbeit an den Acta Borussica. In: Jahrbuch für brandenburgische Landesgeschichte 48 (1997), S. 152-202, hier S. 169f., Nr. 3 (Einstellungserklärung Otto Hintzes, 14. März 1888); zum Ganzen meine in Anm. 28 genannte Studie.

32 Brief Schmollers an Althoff, 24. Oktober 1896, GStA PK, VI. HA, Nachlass Althoff, A II, Nr. 95 II, wohl darauf bezieht sich Hintzes Brief an Schmoller vom 31. Dezember 1896, in dem er von einem auf Befehl Althoffs geschriebenen „Werk“ spricht, das auch publiziert werden solle. 
den könne, eine Absicht, die freilich nicht realisiert worden ist. Politische Dimensionen wurden dann berührt, wenn Hintze - freilich schon in späteren Jahren, um 1900 - im Auftrage Friedrich Althoffs sich zu der Frage gutachterlich zu äußern hatte, ob Wilhelm I. als „der Große“ tituliert werden sollte, wie Wilhelm II. es ja gerne wollte. Hintze wählte den für ihn schon damals sehr typischen methodischen Weg, ,die weltgeschichtliche Anwendung des Regentennamens, der Große““ darzulegen und nach den epochenspezifischen Kriterien zu fragen, nach denen jeweils die „Herrschergröße“ beurteilt worden war, um die Relativität dieser Kategorie im Falle Wilhelms I. herauszustellen. ,Jedenfalls glaube ich weder meine wissenschaftliche Ueberzeugung verleugnen noch den übernommenen Auftrag unausgeführt lassen zu dürfen“. ${ }^{33}$ Hintze formulierte seine Bedenken, verglich - ein heikles Thema im Kaiserreich! - die historische Initiative des alten Monarchen und diejenige Bismarcks und kam zu dem Fazit, dass der Herrscher der Reichsgründungszeit von denen, die den Beinamen „der Große“ etablieren wollten, doch damit ,in eine falsche Pose gebracht ${ }^{\star 34}$ werde - was der Intention des regierenden Monarchen schwerlich entsprach. Für politische Kampagnen schien Hintze nicht der rechte Mann zu sein. Das gab Schmoller Althoff bei anderer Gelegenheit zu bedenken: „er ist auch zu sehr Historiker; in solchen Fragen kann man nur Leute brauchen, welche die stärkste Empfindung für die praktische Politik des Tages haben“. ${ }^{35}$

Und trotzdem strahlte die amtliche Gnadensonne weiter, wichtig zumal in den Jahren nach Hintzes Habilitation 1895, der sein zweites Buch, das über die Seidenindustrie (nicht nur!) im Preußen Friedrichs des Großen, zu Grunde lag. Die Schriften gerade dieser frühen Zeit stehen in einer interessanten Werkkontinuität zu Hintzes späterem Schaffen - Schriften, die von der wissenschaftsgeschichtlichen Forschung sträflich unterschätzt worden sind. Die preußischen Forschungen sind eingebettet in eine Jahrhunderte umspannende Perspektive (nicht nur) europäischer Wirtschaftsgeschichte,

33 Brief (!) Otto Hintzes an Althoff vom 2. Januar 1902, GStA PK, VI. HA, Nachlass Althoff, C 9; ediert bei Neugebauer: Die wissenschaftlichen Anfänge (wie Anm. 30), S. 545f. Anm. 28; das Gutachten Hintzes, das an anderer Stelle lag, ist inzwischen publiziert: vom Brocke, Bernhard: Über den Beinamen „der Große“ von Alexander dem Großen bis zu Kaiser Wilhelm „dem Großen“. Annotationen zu Otto Hintzes Denkschrift „Die Bezeichnung ,Kaiser Wilhelm der Große“" für Friedrich Althoff (1901). In: Neugebauer, Wolfgang (Hg.): Das Thema „Preußen“ in Wissenschaft und Wissenschaftspolitik des 19. und 20. Jahrhunderts. Berlin 2006, S. 231-267 (= Forschungen zur Brandenburgischen und Preußischen Geschichte, Neue Folge, Beiheft 8), die Quelle: S. 256-266; dort die folgenden Mitteilungen S. 264f.

34 vom Brocke: Über den Beinamen (wie Anm. 33), S. 265.

35 GStA PK, VI. HA, Nachlass Althoff, C 12, Bd. 3, Brief Schmollers vom 26. Dezember 1901. 
die zu Hintzes Studien zum „modernen Kapitalismus“ in den 1920er Jahren den Bogen schlagen. In begleitenden Untersuchungen wird die Organisation gesellschaftlicher Interessen im Prozess der Gewerbeentwicklung und Frühindustrialisierung zum Thema, und die Kategorie des Staates, deren Verhältnis zu der der Gesellschaft den Kandidaten Hintze 1883/84 beschäftigt hatte, beginnt schon bisweilen relativ $\mathrm{zu}$ werden und zu verblassen. ${ }^{36}$

Dieser Befund ist deshalb von mehr als nur biographischer Bedeutung, weil damit ein Schlaglicht auf die wissenschaftlichen Denkwelten in einer Epoche geworfen wird, in der die Kategorien von Staat und Nation durchaus nicht immer absolut gesetzt worden sind. Zur mündlichen Habilitationsleistung schlug Hintze dann 1895 vor, am besten über „Karl Marx und die Geschichtsauffassung des Socialismus“ vorzutragen,${ }^{37}$ was die Fakultät jedoch zugunsten eines konventionelleren Themas abgelehnt hat.

Bei der Meldung zur Habilitation hat Hintze in seine weiteren Perspektiven insofern Einblick gewährt, als er erstmals seine weit über Preußen hinausweisenden vergleichend rechts- und strukturgeschichtlichen Interessen zum Programm erhob. ${ }^{38}$ Wenig später tauchte erstmals sein zweites Hauptwerk als Projektidee auf. Aus der unlängst vorgelegten Edition neuer „Briefe und Dokumente“ Friedrich Meineckes ist zu entnehmen, dass Otto Hintze schon 1898 für ein monographisch angelegtes Handbuch eine „systematische, Politik auf historischer Grundlage ““ verfassen wollte, ${ }^{39}$ die als Summe seiner vergleichenden Forschungen also schon seit seinen Privatdozentenjahren neben die „Allgemeine Verfassungsgeschichte der Neueren Staaten“ treten sollte. In dieser würde die Individualität der Entwicklungen stärker dominieren.

Und zunächst war ja gute Zeit für zukunftsweisende Programme. Denn während, aus Ursachen, die in den Universitätsstrukturen begründet waren, ein normaler Privatdozent im Kaiserreich immer länger auf eine besoldete Stelle warten musste ${ }^{40}$ und

36 Alles Weitere mit Belegen bei Neugebauer: Otto Hintzes Weg (wie Anm. 1), bei Anm. 24-31.

37 Humboldt-Universität zu Berlin, Archiv, „Acta [...] betreffend Habilitationen der Privat Docenten vom 1. Februar 1895 bis 6. Februar 1897“, Phil. Fak. Nr. 1221.

38 In der Akte wie Anm. 37; vgl. dazu Neugebauer: Otto Hintze und seine Konzeption (wie Anm. 10), S. 39.

39 Meinecke, Friedrich: Neue Briefe und Dokumente, hg. von Bock, Gisela/Ritter, Gerhard A. München 2012, S. 126 Anm. 4, zu Nr. 31 (vom 30. August 1898), ferner S. 129 Nr. 33 (22. Januar 1899); zur Verfassungsgeschichte meinen oben in Anm. 10 nachgewiesenen Beitrag.

40 Ringer: Das gesellschaftliche Profil (wie Anm. 21), S. 95; und Szöllösi-Janze, Margit: Lebensgeschichte - Wissenschaftsgeschichte. Vom Nutzen der Biographie für die 
geraume Zeit etwa auf privates Vermögen angewiesen blieb, hat Hintze neben dem harten Dienst für den akademischen Editionsbetrieb schon sehr, ja ungewöhnlich schnell universitäre Perspektiven eröffnet bekommen. Die Initiative ging dabei ganz auffälligerweise vom preußischen Kultusministerium aus, formell im Namen des Ministers. „Es ist meine Absicht“, so erfuhr die Philosophische Fakultät in Berlin im Mai 1899, „den Privatdozenten an der philosophischen Fakultät der hiesigen Universität Dr. Otto Hintze zum außerordentlichen Professor zu befördern. Die philosophische Fakultät ersuche ich um eine baldige Aeußerung, ob dortseits Bedenken dagegen obwalten“. ${ }^{41}$ Alles Weitere war in wenigen Wochen erledigt; Hintze wurde mit Bestallung vom 18. August 1899 zum außerordentlichen Professor an der Friedrich-Wilhelms-Universität zu Berlin ernannt, mit dem Auftrag, ,die Wirtschaftgeschichte, die Verfassungs- und Verwaltungsgeschichte und die Politik in Vorlesungen und Uebungen zu vertreten“. ${ }^{42}$ Hintze hatte diese Denomination gewünscht. Seinen Dank adressierte er an Schmoller und an Friedrich Althoff.

Hintze hatte also nicht eine Professur für preußische Geschichte inne, wie er auch nie amtlich zum Historiographen des preußischen Staats oder zum brandenburgischen Historiographen ernannt worden ist; letztere Würde hatte Gustav Schmoller inne, erstere Reinhold Koser, der zu den universitären Lehrern Hintzes gehört hatte. ${ }^{43}$ Wir werden dieser personellen Konstellation aus Hintzes Aufstiegsjahren noch einmal begegnen.

Wenn es ein Zäsurjahr in Leben und Werk Otto Hintzes gegeben hat, so war es das Jahr 1900. Das Dreiecksverhältnis Althoff - Schmoller - Hintze, das seit mehr als zehn Jahren bestand, erklärt es auch, dass wenig später Hintzes immer noch unsi-

Geschichtswissenschaft und Wissenschaftsgeschichte. In: Berichte zur Wissenschaftsgeschichte 23 (2000), S. 17-35, hier S. 24f., S. 34 mit Anm. 39.

41 Humboldt-Universität zu Berlin, Archiv, Phil. Fak. Nr. 1463, Erlass des preußischen Kultusministeriums an die Fakultät, Ausf. i. A. gez. Naumann, Berlin 19. Mai 1899, zu Naumann vgl. Lüdecke: Die Preußischen Kultusminister (wie Anm. 29), S. $31 f$.

42 Humboldt-Universität zu Berlin, Archiv, Phil. Fak. Nr. 1463, Erlass des Unterstaatssekretärs von Bartsch an die Fakultät, 18. August 1899, summarisch Simon, Christian: Staat und Geschichtswissenschaft in Deutschland und Frankreich 1871-1914. Situation und Werk von Geschichtsprofessoren an den Universitäten Berlin, München, Paris. Bd. 1, Bern u. a. 1988, S. 121. Dass der Lehrauftrag Hintzes eigenem Wunsch entsprach, ergibt sich aus seinen Dankschreiben an Schmoller: GStA PK, VI. HA, Nachlass Schmoller, Nr. 156, vom 7. September 1899, und an Althoff: GStA PK, VI. HA, Nachlass Althoff B, Nr. 72, vom 18. September 1899.

43 Neugebauer, Wolfgang: Die preußischen Staatshistoriographen des 19. und 20. Jahrhunderts. In: Ders. (Hg.): Das Thema „Preußen“ (wie Anm. 33), S. 17-60, hier S. 54f. mit weiterer Lit. S. 22; Lehrerverhältnis: Hintze: König Wilhelm (wie Anm. 24), S. 34. 
chere soziale Stellung zwischen Akademie und Universität durch die Übertragung eines „etatmäßigen Extraordinariats“ stabilisiert und dabei - das ist das Wichtige ihm von Althoff ein weiterer, zusätzlicher Lehrauftrag erteilt worden ist. Fortan wurde Hintze ,zugleich [...] noch verpflichtet, auch die specielle Staatenkunde unter Berücksichtigung der Seeinteressen in regelmäßigen Vorlesungen zu vertreten“. ${ }^{44}$ Im Laufe des Wegs zum Ordinariat kam dann auch noch der Spezialauftrag für die niederländische Geschichte hinzu.

Im Hintergrund stand bei alledem die politische und die wissenschaftspolitische Akzentuierung imperialer Strategien, aber zu einem politischen Professor, gar zu einem Aktivisten der zeittypischen Flottenpropaganda ist Hintze nie geworden. ${ }^{45}$ Stattdessen weitete sich seine Perspektive sofort um und nach $1900 \mathrm{zu}$ einer globalen Analytik, wie eine werkgeschichtliche Betrachtung (unter Einbeziehung ungedruckter Quellen) zeigen würde. ${ }^{46}$ Hintze, der sich mit universalgeschichtlichen Programmen und typologisch-vergleichenden Verfahren beschäftigte, ergriff nun die Kategorie der Globalität, der seegestützten Zukunft politischer Strukturen, ganz wesentlich bestimmt von imperialer Beherrschung weltweiter Kommunikation.

An dieser Stelle aber geht es um die Frage, wie die sozialtypologische Stellung des Aufsteigers Otto Hintze sein wissenschaftliches Werk weiter formierte.

Sie hat es entscheidend deformiert. Denn die Konstellation der Kerngruppe Althoff Schmoller - Hintze förderte und band Hintze zugleich. Der soziale Aufstieg war zu bezahlen, durch objektive Abhängigkeiten des nun nicht mehr ganz jungen Gelehrten, vielleicht auch durch subjektive, durch Loyalitäten, von denen sich ein Hintze nicht befreite. $\mathrm{Zu}$ den neun Bänden der Acta Borussica, die von Hintze stammten, traten Zumutungen Schmollers, in eben den Jahren, als Hintzes monographisches Doppelprogramm entstand, das in Aufsatzstudien und breiter Universitätslehre grundgelegt wurde. ${ }^{47}$ Schmoller hatte 1898 eine umfangreiche biographische Monographieserie über „Politiker und Nationalökonomen“ angeregt und mit dem Verlag Frommann in Stuttgart vereinbart; ein großes Vorhaben, das - es sei hier nur angedeutet - Otto Hintze und Max Weber einmal in direkten Kontakt brachte. Aus der

44 Erlass des Kultusministeriums, i. A. gez. Althoff. 18. August 1900, GStA PK, I. HA, Rep. $76 \mathrm{~V}^{\text {a }}$ Sekt. 2, Tit. IV, Nr. 61, Vol. 10; Humboldt-Universität zu Berlin, Archiv, Phil. Fak. Nr. 1463, und Personalakte Lit. H 332, Bd. 1; Weg zum Ordinariat (1902/07): Neugebauer: Die wissenschaftlichen Anfänge (wie Anm. 30), S. 550f. Z. B. McGlelland, Charles E.: The German Historians and England. A Study in Nineteenth Century Views. Cambridge 1971, S. 205.

46 Wie Anm. 1.

47 Vorläufig Neugebauer: Otto Hintze und seine Konzeption (wie Anm. 10), S. 40ff. und passim. 
Reihe ist dann nicht viel geworden, nur zwei Bände sind überhaupt erschienen, darunter im Jahre 1904 die erste Auflage von Hermann Onckens berühmtem Buch über Ferdinand Lassalle. ${ }^{48}$ Hintze hatte nicht nur die ganze Mitarbeiterkorrespondenz zu führen (de facto war er der Redakteur), sondern ihm war auch eine Monographie zu Friedrich II. übertragen worden, an der er rund zehn Jahre lang schwer getragen hat und die trotz starker Investitionen an Kraft und Zeit nie erschienen ist. ${ }^{49}$ Das Ganze war ihm, der sich längst doch ganz andere Ziele gesteckt hatte, sichtbar eine Qual. Mit mehr eigenem Interesse war Hintze an die Biographie des liberalen Politikers und späteren preußischen Finanzministers Johannes von Miquel gegangen, der in jungen Jahren mit Marx in Kontakt gestanden hatte, nicht der einzige damals heikle Punkt im Leben dieser in der Tat interessanten Gestalt, gestorben 1901. Mit Schmoller war Miquel persönlich gut bekannt gewesen. ${ }^{50}$ Unmittelbar nach dessen Tod taucht in Berlin der Gedanke zu einer auf Miquels Nachlass gestützten Biographie des Ministers auf, ein Projekt, für das die Familie Miquel jetzt und in den folgenden Jahrzehnten ein waches Auge und eine steuernde Hand hatte. Dazu kamen die Arbeiten für die Akademie, überdurchschnittlich viele Lehrverpflichtungen, immer wieder Vortragsaufforderungen von Friedrich Althoff und - um nur das noch zu nennen - die alleinige Herausgabe der wichtigsten Fachzeitschrift des preußischen Arbeitsfeldes, der im Hintergrund das wissenschaftliche Reich Gustav Schmollers deckenden „Forschungen zur Brandenburgischen und Preußischen Geschichte ${ }^{\text {“51. }}$.

48 Oncken, Hermann: Lassalle. 1. Aufl., Stuttgart 1904 (= Politiker und Nationalökonomen. Eine Sammlung biographischer System- und Charakterschilderungen, hg. von G. Schmoller und O. Hintze); die 2. Aufl. mit derselben Reihenangabe: Stuttgart 1912; als Bd. 1 der Reihe erschien noch: Fester, Richard: Machiavelli. Stuttgart 1900. Festers Band ist ein Plan der gesamten Reihe beigedruckt, in dem Hintzes „Friedrich d. Gr.“ angekündigt wird und ein Buch Schmollers über Friedrich Wilhelm I.

Das wird in verschiedenen privaten Korrespondenzen Hintzes aus der Zeit 1897-1907 deutlich; Beispiel: an Friedrich Meinecke am 2. Juli 1902, GStA PK, VI. HA, Nachlass Meinecke, Nr. 15.

50 vom Bruch, Rüdiger: Die Staatswissenschaftliche Gesellschaft. Bestimmungsfaktoren, Voraussetzungen und Grundzüge ihrer Entwicklung 1883-1919. In: Hundert Jahre Staatswissenschaftliche Gesellschaft zu Berlin 1883-1983. Berlin 1983, S. 9-69, hier S. 27; GStA PK, VI. HA, Korrespondenzen: Nr. 187 u. Nr. 189.

51 Zum Wissenschaftsimperium Schmollers und speziell zu diesem Publikationsorgan Neugebauer, Wolfgang: Die „Schmoller-Connection“. Acta Borussica, wissenschaftlicher Großbetrieb im Kaiserreich und das Beziehungsgeflecht Gustav Schmollers. In: Kloosterhuis, Jürgen (Hg.): Archivarbeit für Preußen. Symposion der Preußischen Historischen Kommission und des Geheimen Staatsarchivs Preußischer Kulturbesitz aus Anlaß der 400. Wiederkehr der Begründung seiner archivischen Tradition. Berlin 2000, S. 261-301 
Und Hintzes Gesundheit war ja nie die festeste gewesen. Kein Wunder, dass mit der neuen Zusatzlast einer Miquel-Biographie, die Hintze nun erstmals in Probleme und Quellenmassen des 19. Jahrhunderts hineinzog, „der Kessel überheizt“" wurde, sodass er 1902 hatte "schon einmal stoppen müssen“. ${ }^{52}$ Die Arbeiten an diesem Thema der damaligen preußisch-deutschen Zeitgeschichte haben Hintze jahrelang, im Kern bis 1907, aber noch weiter bis 1914, stark, wenn auch ohne Publikationsertrag, beschäftigt, und auch dabei scheint Friedrich Althoff im Hintergrund eine wichtige Rolle gespielt zu haben. Aus einem Schreiben Hintzes vom Jahre 1924 geht jedenfalls hervor, dass am Anfang dieser Forschungen Althoff interveniert und einen wichtigen Quellenbestand in Hintzes Hand gelegt hatte, wo er bis dahin verblieb. ${ }^{53}$ Für unsere Fragestellung genügt der Befund, dass Hintze selbst in seinen besten Jahren im Einsatz seiner Kräfte durchaus nicht frei war, sondern gebunden blieb an die übermächtige Personalkonstellation, die ihn gefördert hatte und aufsteigen ließ im gesellschaftlichen Kräftefeld des kaiserreichlichen Berlin.

Es war nur eine kurze Zeit, in der er um 1908 und 1909 die Gewichte vorsichtig verlagern konnte ${ }^{54}$ in die Richtung des eigenen wissenschaftlichen Wollens, wie er es 1895/98 hatte erkennen lassen. Die preußische Geschichte war für Hintze das Instrument gewesen, um als sozialer Aufsteiger, protegiert von Wissenschaftsorganisatoren aus Ministerium und Gelehrtenwelt, zum akademischen Erfolg zu gelangen. Und die preußische Geschichte wurde nun auch Otto Hintzes Unglück. Es war eine persönliche Katastrophe, die in Gestalt des Hohenzollernjubiläums 1915 - fünf-

(= Veröffentlichungen aus den Archiven Preußischer Kulturbesitz, Arbeitsberichte, Bd. 2), hier S. 288f.

52 Hintze an Friedrich Meinecke am 21. Januar 1902, GStA PK, VI. HA, Nachlass Meinecke, Nr. 15, auch zu Problemen mit der Familie Miquel, die die Quellen nicht freigab; zur Arbeit an diesem Thema aber z. B. noch Hintzes Brief vom 12. Dezember 1905 (ebd.), ferner dort Hintzes Briefe vom 29. Januar und 29. Juni 1907.

53 Eigenhändiger Brief Otto Hintzes vom 28. September 1924, Bundesarchiv Koblenz, Nachlass 1478, Wilhelm Mommsen, Nr. 383; vgl. auch Herzfeld, Hans: Johannes von Miquel. Sein Anteil am Aufbau des Deutschen Reiches bis zur Jahrhundertwende. Bd. 1. Detmold 1938, S. IX; ohne Erwähnung Hintzes, aber mit wichtigen Hintergrundinformationen Mommsen, Wilhelm: Zur Biographie Johannes von Miquels. In: Historische Zeitschrift 164 (1941), S. 529-552, hier S. 550f.

54 In den erhaltenen Fragmenten der Allgemeinen Verfassungsgeschichte finden sich immer wieder Beweise für intensive Arbeit im Jahre 1909: GStA PK, VI. HA, Nachlass Otto Hintze (1 Normalkarton umfassend) in Nr. 2, Bd. 1 (Umschlag „Sardinien 17. 18. Jhdt“), Nr. 2, Bd. 4 (Florenz), Nr. 2, Bd. 6 (Sizilien), Nr. 2, Bd. 7, ferner in Nr. 4 (Österreich) etwa Einladungen aus diesem Jahr; weitere Details zur Werkgeschichte bei Neugebauer: Otto Hintzes Konzeption (wie Anm. 10), S. 47f., S. 73f. 
hundert Jahre regierte nun die Dynastie in der Mark - Hintzes Lebenswerk und sein Programm praktisch zerstörte. Schmoller, Wissenschaftsimperialist nicht nur, aber gerade auf preußischem Arbeitsfelde, ${ }^{55}$ bereitete einen kleinen Feldzug vor, um das Ereignis und damit das Thema endgültig und unter Marginalisierung der Konkurrenz zu besetzen. Immer hatte Otto Hintze es abgelehnt, wenn ihm in den Jahren zuvor das Angebot gemacht worden war, auf der Basis seiner in der Tat einzigartigen, in zwei Jahrzehnten bei Akademiearbeiten erworbenen (Akten-)Kenntnisse doch eine preußische Geschichte im Überblick zu schreiben. Und nun, zur Jahreswende 1909/10, holte Otto Hintze eben die Konstellation der letzten zwei Jahrzehnte ein, die seinen Weg gebahnt hatte: Das Kultusministerium - nun nicht mehr in Person des verstorbenen Friedrich Althoff, sondern dessen langjähriger Adlatus, Rat Schmidt(-Ott) -, Gustav Schmoller und zur Verstärkung Hintzes Lehrer aus Berliner Studienjahren, Reinhold Koser, berieten, wie und von wem die große Gesamtdarstellung der preußisch-brandenburgischen Hohenzollern für das Jahr 1915 geschrieben werden solle, auf wissenschaftliche Art, aber doch für die im gebildeten Publikum, die um Geschichte wussten. ${ }^{56}$ Am Abend des 27. Februars 1910 ist es dann passiert, in einem der Berliner Wissenschaftlersalons im feudalen Berliner Westen, am Kurfürstendamm. Hören wir Gustav von Schmoller, wie er - ungewöhnlich euphorisiert - an diesem Tage „11 Uhr Abends“, rasch zurückgekehrt in seine Villa in der nahen Wormser Straße, dem Geheimrat Schmidt-Ott (dem späteren preußischen Kultusminister) den Vorgang beschreibt: „Ich komme eben aus einer Gesellschaft [...] wo Koser u. ich die Gelegenheit wahrnahmen, in einer Ecke Hintze zu bearbeiten. [...] So scheint mir keine Schwierigkeit mehr vorzuliegen, daß Hintze dem Kaiser vorgeschlagen wird. [...] Hintze ist monarchisch u. konservativ [.] Seine historischen Ideale sind die preußischen". ${ }^{57}$

Immer wieder hatte Hintze im Zuge seines weiten wissenschaftlichen und sozialen Weges neue, fremdgesetzte Aufgaben übernehmen müssen, mal produktive mit Blick auf globale maritime Strukturen und mal nur belastende Buchprojekte, die dann nicht erschienen. Nun aber lag der Zieltermin fest. Wie Schmoller und Koser (im

55 Mit anderer Lit. Neugebauer: Die „Schmoller-Connection“ (wie Anm. 51), passim.

56 Kurz angedeutet bei Schmoller, Gustav von: Fünfhundert Jahre Hohenzollernherrschaft. In: Schmollers Jahrbuch für Gesetzgebung, Verwaltung und Volkswirtschaft im Deutschen Reiche 40 (1916), S. 1-19, hier S. 1.

57 Eigenhändiges Schreiben Schmollers (an Schmidt-Ott), mit der Anrede: „Hochverehrter Herr Geheimrath“, GStA PK, I. HA, Rep. 76 Vc Sekt. 1, Tit. 11, Teil VB, Nr. 50; es handelte sich um den Salon Ernst von Meiers, dazu Neugebauer: Die „SchmollerConnection“ (wie Anm. 51), S. 296. Den Einzelheiten zu Genese und Schicksal dieses (inzwischen mehrmals nachgedruckten) Werkes wird mit Nachweis der Spezialliteratur an anderer Stelle nachgegangen werden. 
Bündnis mit Ministerium und dem Audienz gewährenden Monarchen) Hintze bis in Einzelheiten seiner großen, bis heute sehr lesenswerten Darstellung ${ }^{58}$ (ein-)gebunden haben, wie das Werk nach Erscheinen im Krieg zum inneren Politikum wurde, wie die Rezensenten den Autor behandelten - das gehört nicht hierher. Für Hintze war das Buch, kein Panegyrikus der Hohenzollern, ein Unglück, und das in vielfacher Hinsicht. Denn es brachte sein eigentliches Lebensprogramm nun endgültig zum Einsturz.

„Eine neue Aufgabe habe ich mit gemischten Gefühlen übernommen: Das Jubiläumswerk für 1915“, so berichtete Hintze im Mai 1910 seinem damals in Freiburg lehrenden Freunde Friedrich Meinecke. Es solle „weder Siegesallee noch Bilderbuch“ werden, „sondern eine gemeinverständliche preuß[ische] Geschichte. Koser und Schmoller haben mir so dringend zugeredet, daß ich mich der Aufgabe nicht entziehen konnte, und schließlich ließ mir S. M. selbst unvermutet ankündigen, daß er mich empfangen wolle und ich habe - Koser war auch dabei - am 30. März [1910] eine 5/4stündige Unterredung mit ihm gehabt, wobei er ", Wilhelm II., „nichts geäußert hat[,] was mir als Hindernis erschienen wäre. - Natürlich habe ich mir völlige wissenschaftliche Freiheit ausgemacht", so berichtete Hintze, der Kosers und Schmollers starke Vorgaben dabei unerwähnt ließ. „Hoffentlich gehts gut““. ${ }^{59}$

Es ging nicht gut. Hintze hat die Überlast seines Aufstiegs nicht ertragen. „Ich habe in den letzten Jahren die Arbeit überhaupt übertrieben, weil ich vor Inangriffnahme der Preußischen Geschichte gerne mit dem Bande über allgemeine Verfassungsgeschichte fertig werden wollte, was dann leider doch nicht möglich war", so schreibt Hintze an Schmoller im August 1912. „Ich habe in den Ferien 1910 wochenlang von 9-5 auf dem historischen Seminar ununterbrochen gelesen und excerpirt, ich merkte damals schon, daß die Augen litten“. ${ }^{60}$ Andere Krankheiten traten hinzu, aber im Kern war es der Zusammenbruch von Hintzes Sehkraft, mit der er zahlte und die ihn bald nach dem Krieg zur Emeritierung mit noch nicht 60 Jahren zwang. „Mit meinen Augen bin ich allerdings recht unzufrieden“, so berichtete er im September 1911, trotz kompetenter Behandlung werde es immer schlechter, sodass „die Augen, namentlich das linke, bei längerer Anstrengung ganz versagen. Ich bin

58 Hintze, Otto: Die Hohenzollern und ihr Werk. Fünfhundert Jahre vaterländischer Geschichte. (1. Aufl.) Berlin 1915, zur Vorgeschichte nur ganz undeutlich das Vorwort S. V-VII, wonach das Buch bei Ausbruch des Krieges „schon im Satz“war.

59 GStA PK, VI. HA, Nachlass Meinecke, Nr. 15, Briefkarte Hintzes vom 6. Mai 1910; am 2. Januar 1912 fügt Hintze (ebda.) hinzu: „Jetzt würd’ ich diese Sache nicht mehr übernehmen".

60 Eigenhändiger Brief Hintzes an Schmoller, dat. Liebenstein, 28. August 1912, GStA PK, VI. HA, Nachlass Schmoller, Nr. 204 b. 
fast so übel dran wie“ der Nationalökonom und Finanzwissenschaftler „A. Wagner mit seinen 75 Jahren“. ${ }^{61}$ Ohnmachtsanfälle, kurze Bewusstlosigkeiten des gerade einmal Fünfzigjährigen - er berichtete es an Schmoller - änderten an seiner beruflichen Lage gar nichts, Herzprobleme traten auf, die ,auf langjährige Ueberanstrengung“ zurückgeführt wurden. Die Ärzte warnten ,dringend vor einem Uebermaß an Arbeit; aber wie soll unsereiner mit dem, was als Normalmaß gelten mag, zu irgend erheblichen Leistungen kommen“. ${ }^{62}$ Es blieb der doppelte Druck: der aus der von seinem Mentor auferlegten Last und der aus dem eigentlichen, dem eigenen wissenschaftlichen Wollen. „Es ist nur meine Sorge, ob ich dann das Jubiläumsbuch rasch genug werde fördern können. Und meine vergleichende Verfassungsgeschichte [...], die der Welt eigentlich erst zeigen sollte, was ich will, möchte ich auch nicht ganz aus den Augen verlieren“, so schreibt er an Schmoller am Neujahrstag $1912 .^{63}$

Es waren Monate mit Todesgedanken, wie wir aus intimen Quellen wissen, in denen nun der Name seiner künftigen Frau, der mehr als zwanzig Jahre jüngeren Studentin Hedwig Guggenheimer, erstmals auftaucht.

„Ich denke dran: es war ein heißer Tag. Semesterschluß! Sprechstunde will nicht enden. Verdrossen saß ich zwischen Bücherwänden. Ein fader Jüngling schwatzte ins Gelag. Da kam Pandora ... “64

So hat Otto Hintze sehr viel später die Szene des Kennenlernens geschildert, Vorgeschichte der - in Hintzes Berliner Kollegenkreis damals mit erstaunlich viel Verständnis und einiger Sympathie aufgenommenen - Eheschließung mit der Tochter aus großbürgerlich-jüdischem Münchener Hause zum Jahresende $1912 .{ }^{65}$ Es spricht einiges dafür, dass diese „Ehe [...] ihm vielleicht das Leben gerettet“ hat, wie Friedrich Meinecke in seinen Erinnerungen schrieb. ${ }^{66}$ Fortan leben beide mit zwei Studierzimmern und vier Schreibtischen, angekommen im Berliner Westen, Kurfürstendamm $44^{\mathrm{II}}$. Aber Otto Hintzes Gesundheit blieb gebrochen, niemals kehrte

61 Eigenhändiger Brief Hintzes an Herman von Petersdorff vom 24. September 1911, GStA PK, VI. HA, Nachlass Herman von Petersdorff.

62 Brief Hintzes an Schmoller aus dem Kurort Martinsbrunn bei Meran, 28. Dezember 1912, GStA PK, VI. HA, Nachlass Schmoller, Nr. 203 a.

63 GStA PK, VI. HA, Nachlass Schmoller, Nr. 204 c.

64 Undatiertes Gedicht Otto Hintzes für seine Frau Hedwig, gedruckt bei Oestreich (Hg.): Otto Hintze (wie Anm. 13), S. 75, Nr. 37.

65 Statt der neuen, bisweilen schon redundanten Lit. Oestreich, Brigitta: Hedwig und Otto Hintze. Eine biographische Skizze. In: Geschichte und Gesellschaft 11 (1985), S. 397-419, bes. S. 401f.

66 Meinecke, Friedrich: Strassburg - Freiburg - Berlin. 1901-1919. Erinnerungen. 1. Aufl., Stuttgart 1949, S. 155. 
die Leistungskraft aus der Zeit vor 1915 zurück, arbeitsfähig war er nur noch sehr beschränkt, und es folgten Jahre stark reduzierter Produktion. Als Illusion stellte sich heraus, was er zu seinem 60. Geburtstag an Meinecke schrieb: „Ich werde glücklich sein, wenn es mir noch gelingt, die Litterarischen Pläne, die mir vorschweben, auszuführen: eine kurze Geschichte des deutschen Staats- und Gesellschaftslebens in großen Umrissen - eine allgemeine Neuere Verfassungsgeschichte [...] - und eine allgemeine Staats- und Gesellschaftslehre auf historischer Grundlage, alles Niederschläge aus meinen Vorlesungen ${ }^{[67]}$, allerdings in wesentlich anderer Perspektive und Einstellung, als sie ursprünglich konzipiert waren" ${ }^{68}{ }^{6}$ Hintze hat auch in den folgenden Jahren an diesen Werken weitergearbeitet und gefeilt.

Aber diejenigen, die in den Jahrzehnten des Kaiserreichs seine Karriere ermöglicht hatten, sie haben im Ergebnis sein eigentliches wissenschaftliches Werk verhindert. Die Verfassungsgeschichte mag um 1930 zu einem Abschluss gekommen sein, ohne in einer Epoche ganz anderer geschichtspolitischer Präferenzen noch gedruckt zu werden. In einem Aufflackern von Arbeitskraft in der zweiten Hälfte der 1920er Jahre hat Hintze dann in großen Rezensionsabhandlungen moderne Wissenschaftskonzeptionen diskutiert und seine Positionen formuliert. Er hat jene großen, weltgeschichtlichen Analysen zu tausend Jahren Partizipationskultur, zum Typ des Feudalismus nicht nur in Europa, zu Wesen und Wandlung des modernen Staats ganz wesentlich in der Preußischen Akademie der Wissenschaften und in ihren Abhandlungen - vorgelegt. Eine das gedruckte und das ungedruckte Material auswertende Werkanalyse wird zeigen, wie stark die konzeptionellen Kontinuitäten auch über seine Katastrophen, auch die von 1918/20, hinweg waren. ${ }^{69}$ Sehr vieles ist gedanklich bald nach 1900 entstanden oder wenigstens grundgelegt worden, als sein Lebensprogramm für ihn feststand. Die großen Summen der späten Jahre erscheinen

67 Vgl. Anm. 10 und Anm. 11.

68 Otto Hintze an Meinecke, eigenhändig, 30. August 1921, GStA PK, VI. HA, Nachlass Meinecke, Nr. 15; vgl. Oestreich, Gerhard: Otto Hintzes Stellung zu Politikwissenschaft und Soziologie. In: Hintze, Otto: Soziologie und Geschichte. Gesammelte Abhandlungen zur Soziologie, Politik und Theorie der Geschichte, hg. von Oestreich, Gerhard. Göttingen ${ }^{2} 1964$, S. $7 *-67 *$, hier S. $7 *$ f.

69 Auf der Grundlage ersten zugänglichen Mitschriftenmaterials aus Hintzes Vorlesungen und Seminaren meine Veröffentlichung aus dem Jahr 1993/98: Neugebauer: Otto Hintzes Konzeption (wie Anm. 10), S. 46, S. 56, S. 73; dies ließe sich insbesondere am Feudalismus-Thema weiter exemplifizieren; sehr richtig mit Verweis auf eine Publikation Hintzes aus dem Jahre 1902 Borgolte, Michael: Otto Hintzes Lehre vom Feudalismus in kritischen Perspektiven des 20. Jahrhunderts. In: Fryde, Natalie/Monnet, Pierre/Oexle, Otto Gerhard (Hg.): Die Gegenwart des Feudalismus. Göttingen 2002, S. 247-269, hier S. 250f. 
wie Notbergungen zentraler Gedanken und wichtiger Entdeckungen - mehr war nicht mehr möglich -, Thesen, angelegt in einem langfristig erwachsenden Werk. Neu war die Perspektive auf moderne Diktaturen ${ }^{70}$ und Revolutionen. Stets hatte ihn die Wirkung äußerer „Staaten“-Konflikte auf die inneren politischen Strukturen fasziniert. Nun beschäftigten Otto Hintze Revolutionen als Impuls strukturellen Wandels. Gleich nach der Machtergreifung wurde 1933 bei Hedwig und Otto Hintze hausgesucht. Seine gesammelten Materialien über „Revolutionen in der Weltgeschichte" wurden dabei beschlagnahmt. ${ }^{71}$

Otto Hintze ist im April 1940 in Berlin verstorben. In seinem letzten Jahrzehnt waren die Bedingungen dafür, sein Lebenswerk durch die Veröffentlichung großer Summen abzuschließen, schlechter denn je, wenn es auch nicht zutrifft, dass er nach 1933 gar nichts mehr veröffentlicht hätte. Da seine Hauptwerke unpubliziert blieben, waren aber die Bedingungen für die Rezeption seines Euvres von Anfang an stark belastet, vor allem durch die Reduktion auf seine kleineren Abhandlungen, die das sei hier nur noch angedeutet - in problematischen, noch einmal perspektivisch verzerrten Auswahlbänden seit den 1960er Jahren gleichwohl ein internationales Interesse für sein Schaffen wachriefen.

Der tiefere Grund für die - etwa im Vergleich zu Max Weber - sehr viel ungünstigeren Rezeptionsbedingungen lag freilich nicht in seiner letzten Lebensphase. Er war vielmehr angelegt in der sozialtypisch signifikanten Position als akademischer Aufsteiger im kaiserreichlichen Berlin. In seinen besten, gesundheitlich noch nicht gebrochenen Jahren, nach seiner Habilitation und bis zum Hohenzollernbuch, also 1895 bis 1915, wurde Hintze die Kraft zum großen Werk von eben denen genommen, die ihn im Gefüge von Wissenschaftsorganisation und Staatsverwaltung in dieser Epoche „gemacht“ hatten. Seine Kraft wurde zum Beispiel in Buchprojekte gezwungen, die dann nie erschienen, und die Auftragsarbeit zum Preußenjahr 1915 brachte für ihn - Jahre vor dem November 1918 - den physischen Zusammenbruch. Hintze vermochte es nicht, sich in seiner ausgesprochen bürgerlich-kulturaristokratischen Umgebung aus seiner Aufsteigerrolle zu lösen. Sein Beispiel verweist auf die Notwendigkeit, die wissenschaftsgeschichtliche Biographie sozialhistorisch zu kontextualisieren. $^{72}$

70 Vgl. auch die in Anm. 1 angekündigte Studie.

71 Die Quelle aus der Familie Otto Hintzes bei Oestreich (Bearb.): Otto Hintze (wie Anm. 13), S. 233, Nr. 230.

72 Vgl. grundsätzlich Bödeker, Hans Erich: Biographie. Annäherungen an den gegenwärtigen Forschungs- und Diskussionsstand. In: Ders. (Hg.): Biographie schreiben. Göttingen 2003, S. 9-63, hier S. 19f., S. 28 (= Göttinger Gespräche zur Geschichtswissenschaft, 
Bd. 18); Gestrich, Andreas: Einleitung. In: Ders. (Hg.): Biographie - sozialgeschichtlich. Sieben Beiträge. Göttingen 1988, S. 5-28, hier S. 8. 